POS PROCEEDINGS

\title{
NIBLES: an H I census of local SDSS galaxies
}

\author{
W. van Driel* \\ GEPI \& Station de Radioastronomie de Nançay, Observatoire de Paris, 92190 Meudon, France \\ E-mail: wim.vandriel@obspm.fr
}

\section{S. Schneider}

University of Massachusetts, Astronomy Program, Amherst, MA 01003,Amherst, U.S.A.

\section{Lehnert}

GEPI, Observatoire de Paris, 92190 Meudon, France

\section{and the NIBLES Consortium}

Improving our knowledge of the H I Universe will improve our readiness to use the SKA and its Precursors to their full potential. NIBLES (Nançay Interstellar Baryons Legacy Extragalactic Survey) is a targeted survey of 3000 SDSS galaxies in the Local Volume $(900<\mathrm{cz}<12,000 \mathrm{~km} / \mathrm{s})$ that are distributed evenly over their entire range of total stellar mass (absolute z-band magnitude $\mathrm{M}_{z} \sim-13$ to $-24 \mathrm{mag}$, for $\mathrm{H}_{0}=70 \mathrm{~km} \mathrm{~s}^{-1} \mathrm{Mpc}^{-1}$ ). Using mainly the 100-m Nançay Radio Telescope, after 3500 hours of telescope time in the period 2007-2009 it has produced spectra deeper than the blind ALFALFA survey, and it uniformly samples the local galaxy stellar mass distribution. NIBLES will allow us to address a number of outstanding questions in astrophysics, such as: the global baryon budget within the local volume, gas properties as a function of galaxy dynamical mass, stellar mass, and various galaxy morphology parameters. A combination of short initial and longer follow-up observations is expected to yield an overall detection rate of $75 \%$, which appears to be rather independent of galaxy mass and colour - except for the most luminous $\left(M_{z}=-23\right.$ to -24), gas-poor ellipticals. NIBLES is an open collaboration and anyone interested in the science and willing to contribute to the project is welcome to join the score of NIBLErS.

Panoramic Radio Astronomy: Wide-field 1-2 GHz research on galaxy evolution

June 2-5 2009

Groningen, the Netherlands

\footnotetext{
* Speaker.
} 


\section{The origins of NIBLES}

It has never been more important to understand the H I Universe, with planning for the Square Kilometre Array (SKA) well under way, and the construction of its Precursors instruments ASKAP and MeerKAT started. Our current knowledge of the H I properties of galaxies feeds into the science simulations that are preparing the way for the SKA; improving this knowledge will improve the design requirements of the SKA, allowing it to reach its full scientific potential.

Traditionally, the $\mathrm{H}$ I properties of galaxies have been found by observing optically-selected galaxies, with complete samples only available from relatively small catalogues. Blind H I surveys were limited in both area and numbers of sources. In the late 1990s, the 21-cm multibeam instrument at the 64-m Parkes radio telescope initiated an all-sky survey (HIPASS) that paved the way for studies of large samples of galaxies selected by their H I signal, without reference to their optical characteristics. However, it was a relatively shallow survey.

At the same time, optical and infra-red surveys have improved dramatically with the SDSS and 2MASS catalogues going much deeper than their predecessors and covering, respectively, a large fraction of the sky and the entire sky. This means that they not only uncover more distant sources, but also faint nearby sources that would have previously been missed and thus not targeted for $\mathrm{H} \mathrm{I}$ follow-up observations. Targeted surveys are now viable ways of characterising the $\mathrm{H}$ I properties of many sorts of nearby galaxies.

One should not think, however, that the H I Universe can be best observed through either of these techniques - both will fail to characterise certain sorts of galaxy: gas-poor galaxies for blind H I surveys; optically-faint, gas-rich galaxies for targeted surveys. What is required for a holistic view of the H I Universe is complementary surveys following both methodologies.

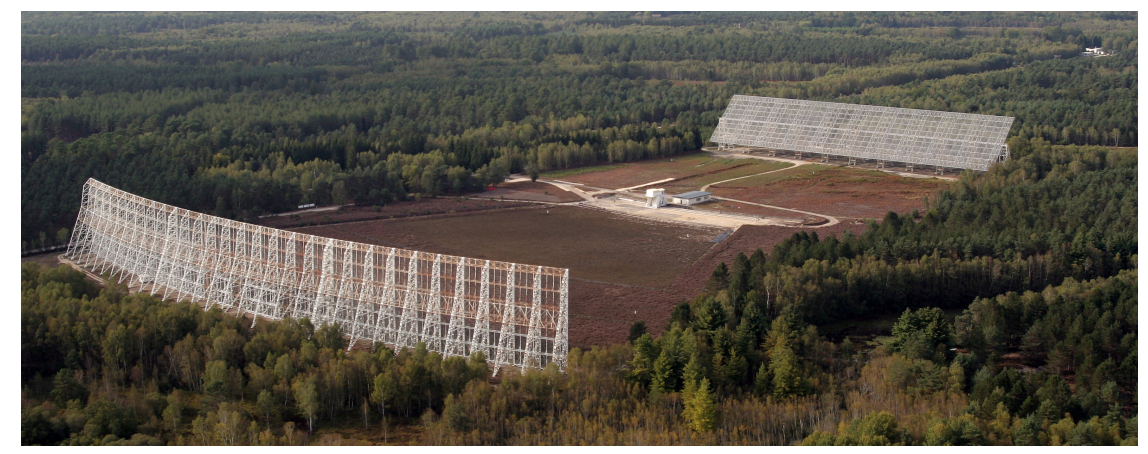

Figure 1: The 100-m class Nançay Radio Telescope (NRT), located in the centre of France, is a meridian telescope of the Kraus/Ohio State design, consisting of a $200 \times 40 \mathrm{~m}$ tiltable flat mirror (right), a $300 \times 35 \mathrm{~m}$ fixed spherical mirror (left), and a focal carriage (centre) moving along a curved rail track. Most sources can be tracked for about 60 minutes. It can observe down to $\delta-38^{\circ}$. Its HPBW is $3.5 \times 23^{\prime}(\alpha \times \delta)$.

For a review on the future of the Nançay decimetric radio telescope (NRT; e.g., Monnier Ragaigne et al. 2003) in 2007, we asked ourselves a number of strategic questions regarding a future NRT H I survey of a large galaxy sample - in particular: (1) can we still learn something new and interesting from such a survey, and (2) why to observe galaxies with a 100m-class telescope, while a $300 \mathrm{~m}$ telescope (Arecibo) is presently doing blind H I galaxy surveys?

The positive answers to both questions are driven by the first-ever optical "blind" galaxy survey, the Sloan Digital Sky Survey (SDSS) which is providing accurate optical photometry and 
redshifts of myriad objects. These data now allow the selection of a robust sample of galaxies in the Local Volume that is based on physical parameters (in our case, total stellar mass), as well as a very time-efficient way to conduct an H I survey of their properties $-50 \%$ of the telescope time can be spent on-source, compared to about only a few percent in a blind H I survey.

These considerations have led to the definition of NIBLES, the Nançay Interstellar Baryons Legacy Extragalactic Survey.

\section{The science goals of NIBLES}

Investigations of the global properties of galaxies are beginning to paint an interesting picture of the history of galaxies. There appears to be a hierarchy of formation as a function of galactic mass - the most massive galaxies likely formed early and violently, while the lower mass galaxies formed later and more quietly. There also appears to be a hierarchy of morphological type, with the densest early-type galaxies forming the earliest while the low density very late type spirals formed the most recently. We broadly know that about $75 \%$ of all stars are in spheroids and about $25 \%$ are in discs. Compared to the stellar mass content, the atomic and molecular gas likely adds a further $20 \%$. The distribution of this gas over morphological type and galaxy mass tells us how efficient the star-formation has likely been as a function of environment, and what this might imply for the evolution of the ensemble of galaxies.

Unfortunately, the error budget for making such estimates is still rather large, even for the local Universe. Looking at the totality of the mass of baryons in the local Universe, one finds there is at least a $25 \%$ uncertainty in the global $\mathrm{H}$ I budget and a $30 \%$ uncertainty in the global $\mathrm{H}_{2}$ budget implying at least a $40 \%$ uncertainty in the total atomic and molecular budget of the local Universe. These phases represent about 20\% of the total baryons outside of stars (but are subject to large possible systematic uncertainties, particularly for lower mass galaxies in which the size of the gas contribution to the baryonic mass is larger and the measurements less certain).

To begin to overcome our ignorance regarding the "how much" and "where is" of atomic (and molecular) gas, we are undertaking NIBLES, a survey of H I at Nançay of 3000 stellar massselected SDSS galaxies in the local $(900<\mathrm{cz}<12,000 \mathrm{~km} / \mathrm{s})$ Universe. For selected objects these data are being complemented by deep Arecibo observations of selected objects not detected by NIBLES, and we intend to obtain $\mathrm{CO}(1-0)$ line observations at IRAM as well, to fill out gaps in the published CO galaxy surveys.

Science goals of NIBLES are:

- Provide a complete and statistically robust analysis of the density of baryons in the local Universe, and determine the phase in which these baryons reside. That is, determine the comoving space density of $\mathrm{HI}$, stellar mass, and dynamical mass. At present, there is at least a $25 \%$ uncertainty in the global $\mathrm{H}$ I budget;

- Determine the H I Mass Function and the joint probability distribution of H I mass/CO luminosity and other galaxy characteristics (i.e., infrared luminosity, morphological type, stellar mass, stellar age, etc.);

- Determine the $\mathrm{HI} / \mathrm{CO}$ gas content and fraction as a function of total stellar mass (fit from the optical/near-infrared spectral energy distribution and $\mathrm{M} / \mathrm{L}_{z}$ ), dynamical mass (estimated from $\mathrm{H}$ I line widths), morphological type, and average stellar density; 
- Determine the systematic relationships and variability between dynamical, stellar, and gas masses (e.g., do the estimated dynamical masses change in proportion to total baryonic mass?);

- Compare galactic gas fractions and metallicity, to determine the effective yields of the largest sample of galaxies available and to allow for the most detailed comparison with theoretical models of the evolution of galaxies. This will enable us to determine the relative influence of outflows and infall on the evolution of galaxies. We will use metallicities determined from the SDSS data and compare them with the overall gas fractions to determine the effective yields as a function of galaxy type, dynamical and stellar masses, and stellar mass surface densities. Comparing these results with chemical evolution models will provide a robust test of our understanding of gas recycling yet performed.

\section{Sample definition and early results}

The Sloan Digital Sky Survey (SDSS) is providing us with an unprecedented rich and complete optical database of local galaxies, which allows a new phase in the census of their gaseous content and other fundamental properties. The 6th data release contains over 56,000 galaxies with radial velocities smaller than $12,000 \mathrm{~km} / \mathrm{s}$. Their absolute z-band ( $\sim 9000)$ magnitudes span a range of over ten magnitudes, $\mathrm{M}_{z} \sim-13$ to -24 (for $\mathrm{H}_{0}=70 \mathrm{~km} \mathrm{~s}^{-1} \mathrm{Mpc}^{-1}$ ), and thus includes galaxies from well above $\mathrm{M}_{*}$ to well below $0.01 \mathrm{M}_{*}$.

NIBLES selection criteria are: (1) high quality SDSS magnitudes, and high quality SDSS optical spectra, (2) within the Local Volume (recession velocity of $900<\mathrm{cz}<12,000 \mathrm{~km} / \mathrm{s}$ ), (3) sampling of each 0.5 magnitude wide bin in $\mathrm{M}_{z}$, with a maximum of 150 galaxies for the most populated bins, and of all galaxies (minimum 20) for the poorer populated bins, (4) focus on the most nearby objects in each $\mathrm{M}_{z}$ bin, as these will have the highest $\mathrm{H}$ I flux densities.

In order to remain all-inclusive in our study of H I properties, we do not want to exclude a priori objects that could be expected to be gas-poor, such as ellipticals and lenticulars - their $\mathrm{H}$ I properties are not well known as a function of total stellar mass (our selection criterion). Objects with $\mathrm{cz}<900 \mathrm{~km} / \mathrm{s}$ are avoided as the SDSS software sometimes splits large diameter galaxies up into multiple sources.

The observing strategy is to obtain intitial, relatively short ( $\sim 35 \mathrm{~min})$ observations of all sources, which have a mean rms noise level of about $2.6 \mathrm{mJy}$ at a velocity resolution of $18 \mathrm{~km} / \mathrm{s}$, followed by longer ( $\sim 50 \mathrm{~min}$ ) observations of all initial non-detections and weak detections, with a peak signal-to-noise ratio of 3.5 or less; the combined long+short integrations have an rms of 1.7 mJy, significantly deeper than the blind ALFALFA survey (Giovanelli et al. 2005). A total of 3250 hours of NRT telescope time has been allocated to this project - 2000 hours in 2007-2008 for the initial, short observations and 1250 hours in 2009 for the longer follow-up observations. A final 400 hours were requested for early 2010 to complete the survey.

Good quality short-integration data were obtained of 2800 galaxies, with an overall detection rate is $56 \%$. In the longer follow-up observations we detected about $50 \%$ of the initial nondetections (with peak $\mathrm{SNR}<2$ ), as well as about two-thirds of the weak initial detections with $2<\mathrm{SNR}<3.5$. This strategy is expected to raise the overall NIBLES H i detection rate (peak $\mathrm{SNR}>3$ ) to about $75 \%$. 


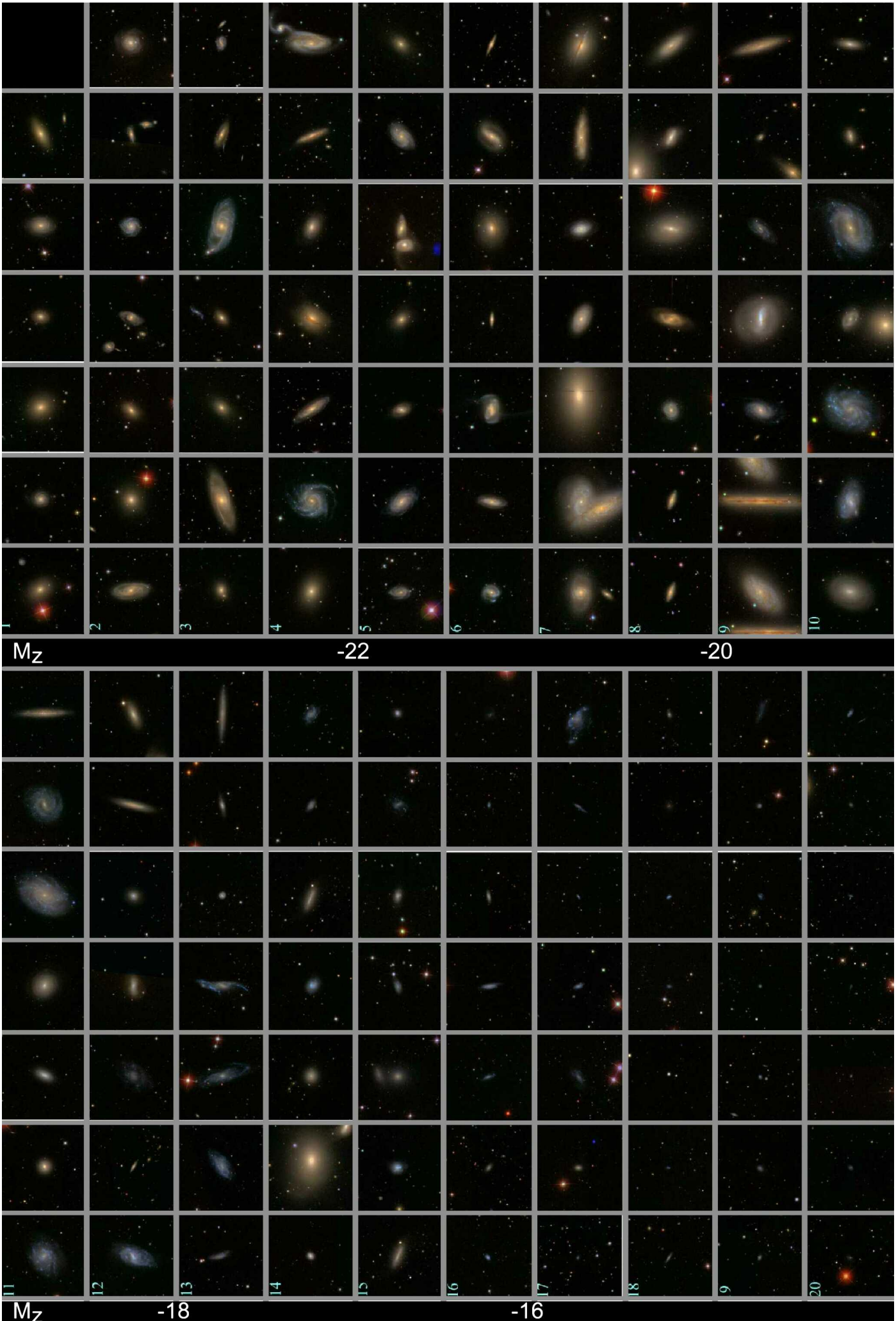

Figure 2: SDSS colour images of some of the first galaxies that were observed in H I for NIBLES. Shown in each vertical row are objects in the first 20 of the 0.5 magnitude wide bins in $\mathrm{M}_{z}$. Each image is $5 \times 5$ arcmin in size. Indicated along the horizontal edges are the bin numbers and absolute z-band magnitudes (total $\mathrm{M}_{z}$ range: -24 to -14 ). 
The initial detection rate is similar over most of the entire magnitude range spanned by the galaxies $\left(\mathrm{M}_{z} \sim-13\right.$ to -23$)$, the exception being the two most luminous bins $\left(\mathrm{M}_{z}=-23\right.$ to -24$)$ dominated by relatively distant, large gas-poor elliptical galaxies. The SDSS has provided us with a large number of remarkably faint and tiny (and H I-rich) nearby galaxies that would simply have been missed in previous optical surveys and thus not targeted for H I follow-up observations, and our deeper observations pick up faint detections missed by less deep blind surveys.

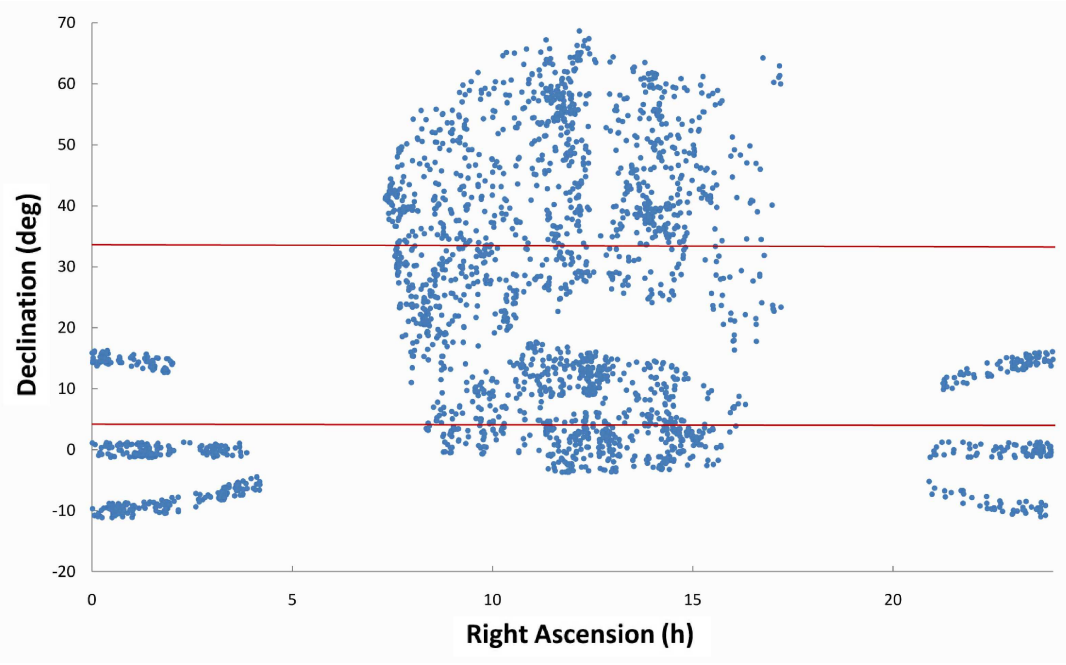

Figure 3: Distribution on the sky of the 2800 NIBLES galaxies of which initial short $(35 \mathrm{~min}) 21 \mathrm{~cm}$ line observations were made. The red horizontal lines indicate the effective declination range of the Arecibo radio telescope $\left(\delta=+3^{\circ}\right.$ to $\left.+33^{\circ}\right) ; 60 \%$ of the sources lie outside the Arecibo range.

The standard deviations per $\mathrm{M}_{z}$ bin of $\mathrm{M}_{H I}$ and $\mathrm{M}_{H I} / \mathrm{L}_{z}$ do not decrease after observing more galaxies, which indicates that these global $\mathrm{H}$ I properties are not determined by a single parameter like $\mathrm{M}_{z}$. The NIBLES detections are relatively common among all colours and luminosities of galaxies, both along the blue and the red sequence, as well as in between. This is surprising since it is thought that galaxies along the "red sequence" in a colour magnitude diagram probably grow through "dry mergers" in which gas is lacking.

A statistical stacking analysis of the NIBLES non-detection spectra is made in the CRUMBS project (Blyth et al., this volume), which confirms our finding that there are significant amounts of "hidden" H I among the initial non-detections - on average, they have an H I mass of $1.2 \cdot 10^{8} M_{\odot}$. This is also consistent with our preliminary analysis of deep (rms $0.9 \mathrm{mJy}$ ) Arecibo observations of 47 initital NIBLES non-detections, of which about $75 \%$ are detected.

\section{References}

[1] Blyth, S., et al. Neutral Hydrogen in Galaxies from Low to High Redshift, this Volume (2009)

[2] Giovanelli, R., Haynes, M.P., Kent, B.R., et al., AJ, 130, 2598 (2005)

[3] Monnier Ragaigne, D., van Driel, W., Schneider, S.E., et al., A\&A, 408 (2003) 